\title{
Neurosis profesional o enfermedad común. Síntomas y estresores
}

\author{
MARCELO TRUCCO B., POLICARPO REBOLLEDO M.
}

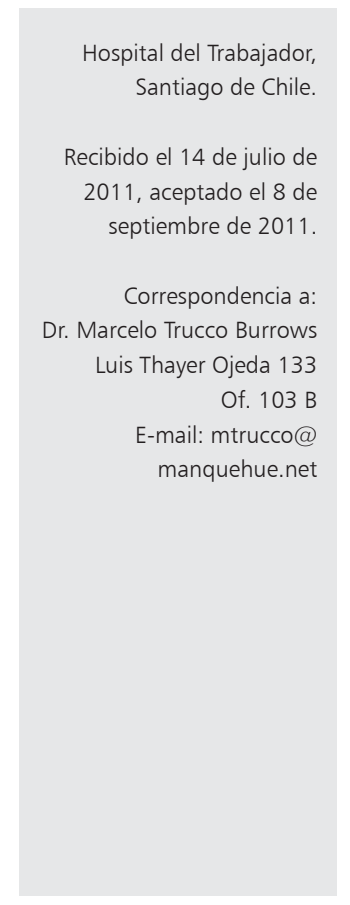

\section{Clinical differences between patients consulting for occupational neurosis and common illnesses}

Background: In Chile, occupational illnesses are defined by the "Work-related accidents and occupational illnesses law". The institutions that administer that law have the legal obligation to determine the "occupational" or "common" origin of accidents and diseases. Aim: To compare symptoms and problems at work in a group of workers consulting for presumptive occupational neurosis (ON), distinguishing cases of OC from cases of common illness (CI). Material and Methods: Two consecutive series of patients were reviewed: 100 cases diagnosed as ON (71\% women) and 200 cases diagnosed as CI (69\% women). The general health questionnaire-30 (GHQ-30) and a questionnaire specially devised at the institution to evaluate ON were applied to participants. Social and demographic features, reasons for consulting and clinical diagnoses were compared in both types of patients. Results: Age, marital status, educational level and type of work were similar among patients with $O N$ and CI. Subjects with CI had a higher frequency of depressive symptoms. The main reason to consult among patients with ON was work overload. The reasons for consulting among patients with CI were interpersonal conflicts and feeling of undervaluation. Conclusions: Patients with CI are predominantly depressive and those with ON have a feeling of work overload.

(Rev Med Chile 2011; 139: 1370-1377).

Key words: Hypochondriasis; Mental health; Occupations.

L os problemas de salud mental son comunes en la población general, estimándose que hasta 1 de cada 5 personas puede presentar síntomas de algún trastorno mental en el curso de un año. Los trastornos mentales dan cuenta de una proporción significativa del ausentismo laboral, y su costo las ubican entre las tres más importantes causas de pérdidas de productividad y de años de vida perdidos por incapacidad ${ }^{1,2}$.

\section{Enfermedad mental y trabajo}

La relación entre trabajo y enfermedades mentales puede plantearse al menos desde dos perspectivas: a) los problemas de las personas que presentan alguna enfermedad mental, que forman parte de la fuerza laboral y los efectos de la enfermedad sobre el desempeño laboral; y b) las enfermedades mentales asociadas a factores de riesgo en el ambiente del trabajo.

Este trabajo pretende abordar el tema de los factores de riesgo percibidos en los lugares de trabajo por los propios trabajadores que consultan por síntomas de trastornos emocionales.

\section{Enfermedad profesional}

El concepto de enfermedad profesional ha sido normado en Chile mediante la Ley $\mathrm{N}^{\circ} 16.744$, de "Accidentes del trabajo y enfermedades profesionales" de 1968. El Decreto Supremo No 109 reglamenta la "calificación y evaluación de los ac- 
cidentes del trabajo y enfermedades profesionales". Su Artículo 161 dice: "para que una enfermedad se considere profesional es indispensable que haya tenido su origen en los trabajos que entrañan el riesgo respectivo, aún cuando éstos no se estén desempeñando a la época del diagnóstico". A su vez, en el Artículo 191, No 13, se establece el correspondiente factor de riesgo: "todos los trabajos que expongan al riesgo de tensión psíquica excesiva y se compruebe relación de causa a efecto". El artículo 19 del D.S. 109 indica que las neurosis profesionales "pueden adquirir distintas formas de presentación clínica, tales como: trastornos de adaptación, trastorno de ansiedad, depresión reactiva, trastorno de somatización y por dolor crónico".

Las mutualidades de empleadores, como administradores de la Ley $\mathrm{N}^{\circ} 16.744$, tienen la responsabilidad legal de calificar el origen "laboral" o "común" de las enfermedades que afectan a los trabajadores de sus empresas afiliadas. En el caso de la Asociación Chilena de Seguridad, ACHS, al Servicio de Salud Mental del Hospital del Trabajador de Santiago, HTS, le corresponde esa responsabilidad en materia de enfermedades profesionales de tipo psiquiátrico, incluyendo evaluaciones psiquiátrica, psicológica, psicométrica y estudio de puestos de trabajo ${ }^{7}$.

\section{Propósito}

El propósito de este estudio es comparar los patrones de síntomas y los problemas laborales más frecuentes de dos muestras de pacientes que consultan por presuntas neurosis profesionales en el Servicio de Salud Mental del Hospital del Trabajador de Santiago (HTS): aquellos que son calificados como de origen laboral ("neurosis profesional") con aquellos que son calificados como "enfermedad común".

\section{Objetivos específicos}

1. Describir y comparar los niveles sintomáticos, mediante los puntajes obtenidos en el General Health Questionnaire, GHQ- 30 (5,6), en una muestra de ambas poblaciones.

2. Comparar los síntomas clínicos más frecuentes en ambos grupos, distinguiendo síntomas predominantemente depresivos y ansiosos.

3. Comparar los diagnósticos clínicos entre ambas muestras.
4. Describir y comparar las principales fuentes de insatisfacción y los problemas percibidos como estresores en el trabajo por los propios trabajadores en ambos grupos.

\section{Método}

Se trata de un estudio retrospectivo sobre una muestra consecutiva de los últimos 100 casos de pacientes calificados de "neurosis profesional" y otros 200 casos consecutivos calificados como de origen común, por el Servicio de Salud Mental del HTS durante el año 2009.

En la atención clínica de ese Servicio de Salud Mental, todos los consultantes son evaluados de acuerdo con un protocolo estandarizado que incluye: examen psiquiátrico y psicológico, estudio psicométrico y estudio de puesto de trabajo. Este último es realizado por psicólogos entrenados, de acuerdo con pautas estandarizadas. Los resultados son analizados en un Comité multiprofesional que decide la calificación definitiva. El proceso de evaluación incluye la administración de dos cuestionarios, una versión traducida y validada en Chile del General Health Questionnaire-30 (GHQ-30), instrumento de tamizaje ampliamente utilizado internacionalmente y validado por este grupo de trabajo en Chile ${ }^{5,6}$, y un Cuestionario Laboral, desarrollado por el Servicio y basado en una batería para el estudio de estrés laboral, validada por un estudio previo (Prieto T. Construcción de un instrumento global para medir stress laboral. Tesis para el Grado de Psicólogo. Universidad Diego Portales, Santiago, 1995) ${ }^{7}$.

El Cuestionario Laboral explora aspectos de satisfacción con el trabajo, esfuerzo, relaciones interpersonales y oportunidades. Consta de 20 ítemes, que se responden en una escala de 1 a 4 puntos, dependiendo del sentido de la afirmación, siendo siempre el mayor puntaje asignado a la situación menos satisfactoria o más estresante. Por ejemplo:

L1. Me impongo metas de rendimiento muy altas: Nunca $=1 ;$ A veces $=2 ;$ A menudo $=3 ;$ Siempre $=4$.

L2. Me agrada el trabajo que desempeño: Nunca $=$ $4 ;$ A veces $=3 ;$ A menudo $=2 ;$ Siempre $=1$.

\section{Muestra}

La población objetivo del presente estudio descriptivo, la constituyen todos los trabajadores 
consultantes en el Servicio de Salud Mental del Hospital del Trabajador de Santiago de la Región Metropolitana en el año 2009 por síntomas de posible enfermedad profesional psiquiátrica, los cuales ascienden a un total de 520, de los cuales 420 fueron calificados en la categoría de Enfermedad Común y 100 como con Neurosis Profesional.

De acuerdo a ello, se estimó un tamaño muestral para el grupo de pacientes con Enfermedad Común a través de Muestreo Aleatorio Simple usando varianza máxima de proporciones, para un $95 \%$ de confianza y un $5 \%$ de error asociado a la estimación, obteniéndose a través de este procedimiento, un tamaño muestral óptimo de 200 pacientes, seleccionados sistemáticamente con partida al azar. Respecto del grupo de pacientes con Neurosis Profesional, se estimó como muestra la selección del total de los casos.

\section{Análisis}

Se realizó análisis descriptivo para la muestra en función de cada una de las variables consideradas en ambos cuestionarios de modo de obtener un panorama general sobre el comportamiento de las variables al interior de la muestra de pacientes estudiados, a partir de tablas de contingencia, gráficos y porcentajes que den cuenta de la distribución de las variables de acuerdo a su clasificación como pacientes con Enfermedad Común y pacientes con Neurosis Profesional.

Se realizaron pruebas no paramétricas de significación estadística para evaluar asociación entre variables categóricas (sexo, estado civil, diagnóstico, etc.) y adicionalmente se evaluó la intensidad de la asociación mediante estadísticos ad hoc.

Para evaluar diferencias de promedios se realizaron pruebas de significación paramétricas (Prueba T y Anova), y pruebas no paramétricas para evaluar diferencias en las formas de distribución de datos (Prueba U de Mann-Whitney y Prueba Kruskal-Wallis).

\section{Resultados}

La muestra, de 300 trabajadores que consultaron por síntomas de presunta "neurosis profesional" durante el año 2009, está compuesta por 200 casos consecutivos calificados de "enfermedad común" y 100 casos consecutivos calificados de "neurosis profesional". En la Tabla 1 se muestran las principales características socio demográficas de ambas muestras: 70 por ciento son mujeres (al contrario de los accidentes del trabajo, en que 70 por ciento son varones). Algunas diferencias que cabe mencionar: la mayor proporción de soltero(a) s en la muestra de "Enfermedad común" (EC) y de casados/convivientes en la de "Neurosis Profesional" (NP); la mayor proporción de personas con estudios superiores en la muestra de NP. Sin embargo, éstas no son significativas.

Los motivos de consulta se han agrupado en tres categorías, según se observa en la Tabla 2. En la muestra de EC predominan los casos de "conflictos interpersonales y sentimientos de subvaloración"

Tabla 1. Datos sociodemográficos de la muestra en porcentajes

\begin{tabular}{|c|c|c|}
\hline & $\begin{array}{l}\text { Enfermedad } \\
\text { común } \\
n=200\end{array}$ & $\begin{array}{l}\text { Neurosis } \\
\text { profesional } \\
n=100\end{array}$ \\
\hline Mujeres & 69,5 & 71,0 \\
\hline Edad: 19 a 29 años & 26,5 & 23,0 \\
\hline 30 a 49 años & 50,5 & 53,0 \\
\hline 50 a 78 años & 23,0 & 24,0 \\
\hline \multicolumn{3}{|l|}{ Estado civil: } \\
\hline Soltero(a) & 37,5 & 26,3 \\
\hline Casado(a)-Conviviente & 49,5 & 61,6 \\
\hline $\begin{array}{l}\text { Separado(a)- } \\
\text { Divorciado(a) }\end{array}$ & 11,0 & 10,1 \\
\hline Viudo(a) & 2,0 & 2,0 \\
\hline \multicolumn{3}{|l|}{ Escolaridad } \\
\hline $\begin{array}{l}\text { Escolaridad obligatoria } \\
\text { incompleta }\end{array}$ & 4,5 & 6,0 \\
\hline $\begin{array}{l}\text { Escolaridad obligatoria } \\
\text { completa }\end{array}$ & 56,5 & 34,0 \\
\hline $\begin{array}{l}\text { Estudios superiores } \\
\text { incompletos }\end{array}$ & 5,5 & 20,0 \\
\hline $\begin{array}{l}\text { Estudios superiores } \\
\text { completos }\end{array}$ & 33,5 & 40,0 \\
\hline \multicolumn{3}{|l|}{ Ocupación } \\
\hline Profesional & 32,0 & 37,4 \\
\hline Técnico & 17,5 & 21,2 \\
\hline Administrativo & 33,0 & 32,3 \\
\hline Servicios & 9,0 & 4,0 \\
\hline Operario & 8,5 & 5,1 \\
\hline
\end{tabular}


Tabla 2. Principales motivos de consulta agrupados

\begin{tabular}{|lcc|}
\hline Motivo de consulta & $\begin{array}{c}\text { Enfermedad } \\
\text { común } \\
\text { n= 200 }\end{array}$ & $\begin{array}{c}\text { Neurosis } \\
\text { profesional } \\
\mathbf{n}=\mathbf{1 0 0}\end{array}$ \\
\hline $\begin{array}{l}\text { Sobrecarga laboral } \\
\begin{array}{l}\text { Conflictos interperso- } \\
\text { nales y sentimientos de } \\
\text { subvaloración ("hostili- } \\
\text { zación") }\end{array}\end{array}$ & $13,5 \%$ & $46,0 \%$ \\
\hline Otros & $14,5 \%$ & $36,0 \%$ \\
\hline
\end{tabular}

y en los de NP, la "sobrecarga". Esa diferencia es significativa $(p<0,001)$. Cabe señalar que en tres casos de NP, el motivo de consulta fue acoso sexual.

El nivel sintomático, como cabe esperar en una muestra de personas que consultan en un servicio de salud mental, es elevado. El puntaje promedio en el GHQ-30 es 22 con una desviación estándar de 6,16 y una mediana de 23, en la muestra total. En Chile se ha estimado el punto de corte para detectar "probables casos" de trastorno emocional en 11/12 (6,8). La Tabla 3 muestra los síntomas más frecuentes según el cuestionario GHQ-30 en el conjunto de los pacientes (registrados por más de la mitad de los casos).

No se encontró asociación significativa de los puntajes del GHQ-30 con género, edad, escolaridad, estado civil, ocupación o motivo de consulta. Sin embargo, los puntajes de la muestra de EC son significativamente mayores que los de los casos de NP (Prueba U de Mann-Whitney).

Desde el punto de vista cualitativo, las diferencias sintomáticas se observan especialmente en el espectro depresivo. La Tabla 4 muestra que los seis síntomas en los cuales existen diferencias significativas de frecuencia son de tipo depresivo y predominan en los casos de EC.

Los diagnósticos clínicos efectuados en el Servicio de Salud Mental, siguiendo las nomenclaturas internacionales, del DSM-IV (Manual diagnóstico y estadístico de los trastornos mentales, de la Asociación Psiquiátrica Americana,1995) y CIE-10 (Clasificación Internacional de Enfermedades $10^{\circ}$ Revisión), se han agrupado en la Tabla 5 . Se encontró diferencias significativas $(\mathrm{p}<0,05)$

Tabla 3. Síntomas más frecuentes en una muestra consecutiva de pacientes calificados de "enfermedad y común" (200) y de "neurosis profesional" (100), según respuestas al GHQ-30

\begin{tabular}{|c|c|c|c|c|}
\hline $\mathbf{N}^{\circ}$ & PREGUNTAS GHQ - 30 & EC \% & NP $\%$ & Todos $\%$ \\
\hline 7 & $\begin{array}{l}\text { ¿Se despierta en la mañana sintiendo que no ha logrado descansar? (mucho } \\
\text { más que de costumbre) }\end{array}$ & 74,5 & 75,8 & 74,9 \\
\hline 1 & ¿Se ha sentido decaído o desganado? (mucho más que de costumbre) & 75,4 & 70,7 & 73,8 \\
\hline 27 & $\begin{array}{l}\text { ¿Se ha sentido nervioso, con el cuerpo tenso y apretado? (mucho más que de } \\
\text { costumbre) }\end{array}$ & 69,4 & 64,3 & 67,7 \\
\hline 6 & $\begin{array}{l}\text { ¿Ha estado despertando muy temprano, sin poder dormirse nuevamente? } \\
\text { (mucho más que de costumbre) }\end{array}$ & 67 & 66 & 66,7 \\
\hline 21 & ¿Se ha sentido triste y deprimido? (mucho más que de costumbre) * & 73,1 & 48,5 & 65 \\
\hline 8 & ¿Se ha desvelado a causa de preocupaciones? (mucho más que de costumbre) & 65,3 & 58 & 62,9 \\
\hline 2 & $\begin{array}{l}\text { ¿Ha tenido dolor o sensación de abombamiento en la cabeza? (mucho más que } \\
\text { de costumbre) }\end{array}$ & 59,8 & 59 & 59,5 \\
\hline 18 & ¿Se ha puesto irritable y mal genio? (mucho más que de costumbre) & 57,4 & 57,1 & 57,3 \\
\hline 10 & ¿Le cuesta quedarse dormido? (mucho más que de costumbre) & 60,5 & 47 & 56 \\
\hline 11 & $\begin{array}{l}\text { ¿Le cuesta permanecer dormido toda la noche, una vez que se duerme? (mu- } \\
\text { cho más que de costumbre) }\end{array}$ & 56,1 & 51 & 54,4 \\
\hline 20 & ¿Ha sentido que sus problemas lo agobian? (mucho más que de costumbre) & 55,8 & 41,4 & 51 \\
\hline 4 & $\begin{array}{l}\text { ¿Ha podido concentrarse bien en lo que hace? (mucho menos que de costum- } \\
\text { bre) }\end{array}$ & 52,3 & 47 & 50,5 \\
\hline
\end{tabular}

* Diferencia significativa entre EC y NP $(p<0,05)$ 


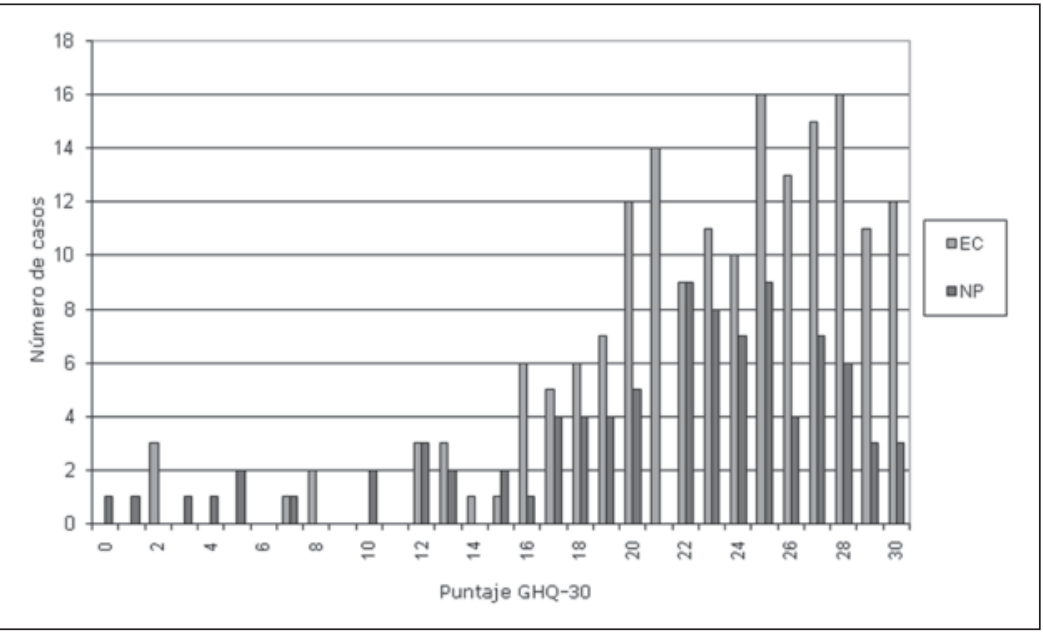

Figura 1. Puntajes del GHQ-30 entre casos de Enfermedad Común (EC) y casos de Neurosis profesional (NP).
Tabla 4. Diferencias significativas en el GHQ 30 entre casos de neurosis profesional y casos de enfermedad común

\begin{tabular}{|lcc|}
\hline & EC \% & NP $\%$ \\
\hline $\begin{array}{l}\text { 13. ¿Ha perdido interés por las cosas que hace? (Mu- } \\
\text { cho más que de costumbre) }\end{array}$ & 52,0 & 32,0 \\
$\begin{array}{l}\text { 17. ¿Ha sentido que no logra sobreponerse en sus } \\
\text { problemas? (Mucho más que de costumbre) }\end{array}$ & 45,4 & 28,9 \\
$\begin{array}{l}\text { 21. ¿Se ha sentido triste y deprimido? (Mucho más } \\
\text { que de costumbre) }\end{array}$ & 73,13 & 48,5 \\
$\begin{array}{l}\text { 23: ¿Ha sentido que usted no vale nada? (Mucho más } \\
\text { que de costumbre) }\end{array}$ & 31,6 & 15,3 \\
$\begin{array}{l}\text { 24. ¿Ha sentido que la vida no ofrece esperanza algu- } \\
\text { na? (Mucho más que de costumbre) }\end{array}$ & 20,9 & 11,1 \\
$\begin{array}{l}\text { 25. ¿Se siente optimista respecto de su futuro perso- } \\
\text { nal? (Mucho menos que de costumbre) }\end{array}$ & 36,7 & 12,2 \\
\hline
\end{tabular}

Tabla 5. Diagnósticos clínicos en casos de enfermedad común y neurosis profesional

\begin{tabular}{|lcc|}
\hline & $\begin{array}{c}\text { Enfermedad } \\
\text { común (EC) }\end{array}$ & $\begin{array}{c}\text { Neurosis } \\
\text { profesional } \\
\text { (NP) }\end{array}$ \\
\hline Trastorno de adaptación ansioso & $31,2 \%$ & $27,0 \%$ \\
\hline Trastorno de adaptación depresivo & $4,5 \%$ & $2,0 \%$ \\
\hline Trastorno de adaptación mixto & $27,6 \%$ & $36,0 \%$ \\
\hline Episodio depresivo mayor & $24,1 \%$ & $19,0 \%$ \\
\hline Otros & $8,0 \%$ & $1,0 \%$ \\
\hline Sin diagnóstico psiquiátrico & $4,5 \%$ & $15,0 \%$ \\
\hline
\end{tabular}

entre las muestras de EC y NP. En el primer grupo los trastornos depresivos mayores, así como los trastornos de adaptación con síntomas depresivos, son significativamente más frecuentes que en el grupo NP. Aquellos pacientes calificados como casos de "neurosis profesional" $y$ "sin diagnóstico psiquiátrico" son aquellos cuyos síntomas se ha estimado que son de origen laboral, sin que se haya especificado otro diagnóstico (15 por ciento del total).

En la aplicación del Cuestionario Laboral los puntajes variaron entre 28 y 75 , con una media de 48,9 , mediana de 49 y desviación estándar de 8,56 . Aplicando prueba $T$, para variables con 2 categorías y Anova, para variables con más de 2 categorías, no se encontró asociación significativa de los puntajes en el Cuestionario Laboral con: calificación de casos en EC o NP, género, edad, escolaridad, estado civil, ocupación y motivo de consulta.

Por otra parte, sí se encontraron diferencias significativas en la frecuencia de respuestas por ítemes entre casos de EC y de NP, como se observa en la Tabla 6. Los trabajadores calificados como NP responden 
Tabla 6. Diferencias significativas en el cuestionario laboral entre casos de enfermedad común y casos de neurosis profesional

\begin{tabular}{|lcc|}
\hline & EC \% & NP \% \\
\hline $\begin{array}{l}\text { 4. Creo que tengo demasiado trabajo, } \\
\text { tanto que no lo puedo completar duran- } \\
\text { te mi jornada (R. A menudo y Siempre) }\end{array}$ & 39,5 & 66,0 \\
$\begin{array}{l}\text { 10. Siento que trabajo apremiado por } \\
\text { el tiempo y los plazos (R. A menudo } \\
\text { y Siempre) }\end{array}$ & 47,7 & 64,3 \\
$\begin{array}{l}\text { 11. Siento que mi trabajo es recono- } \\
\text { cido y valorado (R. Nunca) }\end{array}$ & 48,7 & 36,4 \\
$\begin{array}{l}\text { 19 a. El ambiente humano de mi trabajo } \\
\text { es agradable (R. A menudo y Siempre) }\end{array}$ & 33,5 & 49,5 \\
$\begin{array}{l}\text { 19 b. El ambiente humano de mi tra- } \\
\text { bajo es agradable (R. Nunca) }\end{array}$ & 22,5 & 14,1 \\
\hline
\end{tabular}

con significativamente mayor frecuencia que los casos de EC que tienen exceso de trabajo (ítem 4) y que trabajan bajo apremio de tiempos y plazos (ítem 10); en tanto que los casos de EC responden con significativamente mayor frecuencia que sienten que su trabajo es poco valorado o reconocido (ítem 11) y que el ambiente humano de su trabajo es poco agradable (ítem 19).

\section{Comentario y conclusiones}

En un estudio previo, habíamos descrito algunas características de una muestra de 228 consultantes en este mismo Servicio del Hospital del Trabajador de Santiago, correspondiente a los años 1996 y $1997^{4,8,9}$. Aunque el número de consultas anuales por posibles "neurosis profesionales" ha aumentado año a año desde esa época, la mayoría de los casos siguen siendo calificados de Enfermedad Común: en el estudio previo, 73 por ciento; en 2009,79 por ciento. También han variado ciertas características socio demográficas: la población actual es predominantemente femenina comparada con aquélla muestra anterior (70 versus 51 por ciento); su edad promedio es ligeramente mayor; tiene menos casados/convivientes (53 versus 70 por ciento); e incluye trabajadores con mayor escolaridad y número de profesionales (34 versus 20 por ciento), entre otras. Probablemente éstos reflejan algunos de los cambios ocurridos en nuestra sociedad en las últimas décadas. Los diagnósticos clínicos en ambos estudios son similares: predominan los trastornos de adaptación, con sintomatología ansiosa y mixta (ansiosa y depresiva), seguidos por los trastornos depresivos. Sin embargo, en el estudio anterior, las comparaciones entre NP y EC no fueron hechas con los instrumentos que se han aplicado en los últimos años, por lo que el estudio actual proporciona información más detallada.

Un estudio exploratorio con una muestra de 44 pacientes derivados por el Instituto de Seguridad Laboral, ISL, a la Clínica Psiquiátrica de la Universidad de $\mathrm{Chile}^{10}$, muestra algunas diferencias con éste: casi la mitad es diagnosticado como trastorno depresivo; 84 por ciento son considerados de origen laboral. Las muestras no son enteramente comparables, ya que en ese trabajo se trata de una muestra más selectiva: los pacientes derivados ya son portadores del diagnóstico presuntivo de neurosis profesional; en tanto que en el HTS los pacientes son derivados de las agencias de la ACHS para evaluación, pueden llegar desde las empresas, pueden consultar directamente o son derivados desde las Isapres, donde han sido rechazados. No obstante, los presuntos factores de riesgo informados por los propios pacientes son similares a los que hemos descrito en el presente estudio, pero no encuentran diferencias significativas entre los casos de enfermedad profesional y los de enfermedad común.

El propósito general que nos planteamos fue comparar las dos muestras de trabajadores que consultan por síntomas de trastornos emocionales y que son calificados como de origen laboral ("neurosis profesional") o como "enfermedad común", de acuerdo con las normas legales del sistema de seguridad social chileno. Con ese objetivo a la vista, hemos concluido lo siguiente:

1. Ambos grupos cumplen con los criterios de "casos", en el sentido de presentar síntomas significativos de trastorno emocional, tanto desde el punto de vista clínico como a través de sus respuestas al cuestionario GHQ-30. Los casos de EC son significativamente más sintomáticos que los de NP; y los síntomas que mejor los diferencian son de tipo depresivo, que predominan en los casos de EC.

2. Coincidentemente con lo anterior, si bien los diagnósticos clínicos entre ambos grupos son 
similares, entre los casos de EC se da una frecuencia significativamente mayor de cuadros depresivos, incluyendo depresiones mayores y trastornos adaptativos de tipo depresivo.

3. Un aspecto que se consideró en este estudio fue el motivo principal por el cual las personas consultan, que se debe distinguir del diagnóstico clínico. Así mismo, se debe diferenciar claramente el diagnóstico (acto médico) de la "calificación" (acto administrativo, médicolegal).

4. Nuestros resultados indican que los casos de EC consultan predominantemente por problemas en las relaciones interpersonales, que hemos agrupado en una categoría que denominamos "conflictos interpersonales y sentimientos de subvaloración”. En cambio, el principal motivo de consulta de los casos de NP se asocia a presión de trabajo y "sobrecarga".

5. Los niveles (puntajes) de estresores laborales, medidos por un cuestionario autoadministrado son similarmente altos y no diferencian entre ambos grupos. No obstante, el tipo de estresores en los cuales se encontraron las mayores diferencias, diferencian a los casos de EC, que se sienten subvalorados y perciben a las personas de su ambiente laboral como poco agradables, de los casos de NP, en los cuales predominan la sobre carga cuantitativa de trabajo y las presiones por tiempos y plazos.

Finalmente, podemos concluir que se trata de dos poblaciones similares, con las diferencias descritas. La calificación del "origen laboral" de trastornos emocionales es difícil y se lleva a cabo en virtud de ciertas convenciones y criterios basados en la normativa legal del sistema de seguridad social chileno. Dicha normativa ha cambiado recientemente, ampliando el concepto de "neurosis profesional y haciéndolo más congruente con las clasificaciones clínicas contemporáneas. Sin embargo, mientras persista la obligación de diferenciar y definir el "origen laboral" en oposición a las "enfermedades comunes", en materia de trastornos psiquiátricos seguirá siendo un tema complejo.

Desde la perspectiva de la salud mental de los trabajadores en general, independiente de la calificación médico-legal de los casos, debe llamar la atención el aumento sostenido de las consultas por trastornos mentales atribuidos por las personas al trabajo. En particular, los cuadros depresivos, cuyas consecuencias en los propios enfermos, sus familias, sus empleos, productividad y para la economía del país en general son graves. La literatura reciente aporta pruebas contundentes de la relación entre factores psicosociales identificables en el trabajo y la probabilidad de enfermar de depresión $\mathrm{y}$ otros trastornos mentales relacionados ${ }^{11,12}$.

La promoción de la salud mental y la prevención de los trastornos mentales son tareas de la mayor relevancia para la salud pública y para nuestra sociedad. No se entiende que las empresas y organizaciones, tanto públicas como privadas puedan restarse a la responsabilidad de enfrentar estos problemas. Existen los fundamentos y las tecnologías apropiadas para hacerlo ${ }^{13,14}$.

Agradecimientos: A la Sra. Gabriela Huepe Ortega, quien tuvo a su cargo el análisis estadístico. A la Sra. Rosa Alcayaga, por su rol en la recolección de los datos.

\section{Referencias}

1. Cutcliffe T. Mental illness a burden on productivityBusiness-theage.com.au. June 1, 2007: Consultado: 2102-2009.

2. Eaton WW, Martins SS, Nestadt G, Bienvenu OJ, Clarke D, Alexandre P. The Burden of Mental Disorders. Epidemiol Rev 2008; 30 (1): 1-14; doi:10.1093/epirev/mxn011.

3. Harnois G, Gabriel P. (2000). Mental Health and Work. Impact, Issues and Good Practices. WHO/ILO, Geneva.

4. Oyarzún F, González X, Soto M, Tapia L, Trucco M. Neurosis ocupacional. Boletín Científico ACHS 1999; (1): 53-5.

5. Trucco M, Campusano ME, Larraín S. Un cuestionario para detectar desórdenes emocionales. Estudio de validación preliminar. Rev Chil Neuro-Psiquiat 1979; 17: 20-5.

6. Goldberg DP, Williams P: A user's guide to the General Health questionnaire. Windsor, UK: NFER-Nelson 1988.

7. Trucco M, Valenzuela P. Una batería de cuestionarios para el estudio de estrés ocupacional". Rev Ch NeuroPsiquiat1998; 36: 59-165.

8. Trucco M. El estrés y la salud mental en el trabajo. Ciencia \& Trabajo (ACHS) 2004; 6, 149: 185-8.

9. Trucco M, Rebolledo P, Oyarzún F, Soto M. Neurosis profesional. Rev Chil Neuro-Psiquiat 1998; 36: 149-151.

10. Miranda G, Rojas G. Trastornos mentales de origen laboral: calificación y ponderación de riesgos. Rev Psiq clin 2009; 46: 38-47. 
Neurosis profesional o enfermedad común - M. Trucco et al

11. Netterstrom B, Conrad N, Berch P, Fink P, Olsen O, Rugulies $\mathrm{R}$, et al. The relation between work-related psychosocial factors and the development of depression. Epidemiol Rev 2008; 30: 118-32.

12. Kivimäki M, Vahter J, Kawachi I, Ferrie JE, Oksanen T, Joensuu M, et al. Psychosocial work environment as a risk factor for absence with a psychiatric diagnosis: An instrumental-variables analysis. Am J Epidemiol 2010; 172: 167-72.

13. Trucco M. Promoción de la salud mental de los trabajadores. Rev Psiquiatría (Chile) 1997; 14 (2): 65-71.

14. Trucco M, Horwitz N. Una guía para la promoción de la salud mental en los lugares de trabajo. Boletín Científico ACHS 2003; 9: 23-8. 